\title{
Automatic Organ Validation of B-mode Ultrasound Images for Transmission to Cloud.
}

\author{
R. Bharath, P. Rajalakshmi, \\ Department of Electrical Engineering, \\ Indian Institute of Technology, Hyderebad, \\ email: $\{$ ee13p0007, raji \}@iith.ac.in
}

\begin{abstract}
Miniaturization in size of Medical ultrasound scanning machine made it to use in point of care applications. Lack of sonographers and their unwillingness to work in rural areas limit the benefits of ultrasound system in rural healthcare. Diagnosis of patients through ultrasound is done by visualizing the ultrasound scanned images of organs. Diagnosis through telemedicine involves transmitting of ultrasound images from rural locations to cloud, where sonographer can remotely access the ultrasound data from cloud and generate the report, thus reducing the geographical separation between patients and doctors. Due to lack of adequate sonographers, ultrasound scanning in remote areas is operated by semi-skilled clinicians. Most of the images generated by semi-skilled clinicians are not useful for diagnosis. Transmitting all these images increases the data in cloud, drains the battery of portable ultrasound machine and increases latency in medication. This paper provides automatic B-mode ultrasound image validation based on organ information present in the image for diagnosis, thus avoiding transmission of invalid images to cloud. Linear kernel SVM classifier trained with first order statistic features of image with/without organs is used to classify the images into valid and invalid for diagnosis. The algorithm resulted with a recognition efficiency of $94.2 \%$ in classifying the ultrasound images.
\end{abstract}

Index Terms-Point of care, sonographers, SVM, telemedicine, ultrasound scanning machine.

\section{INTRODUCTION}

Ultrasound medical imaging is a non-invasive imaging modality used to capture images of organs in the body. Ultrasound imaging is considered as safe due to its use of nonradioactive ultrasound waves for image reconstruction. Unlike computer tomography (CT) and magnetic resonance imaging (MRI), ultrasound scanning machines offers real time imaging and needs limited test bench for image acquisition. Ultrasound scanning machine services are limited to well established hospitals due to its high formfactor. Recent advancements in computing platforms like field programmable gate arrays (FPGA) and digital signal processors (DSP) realized the standalone ultrasound machines to handheld level. Handheld ultrasound machines are as flexible as mobile phones and can be used for point of care (POC) applications, POC includes battle fields, remote areas, ambulance etc. Handheld ultrasound machine of GE vscan is shown in Fig. 1.

In past, several portable ultrasound machines have been developed with various features to offer better healthcare services. Ultrasound imaging system based on smart phone is discussed in [1]. A PC based ultrasound imaging system is

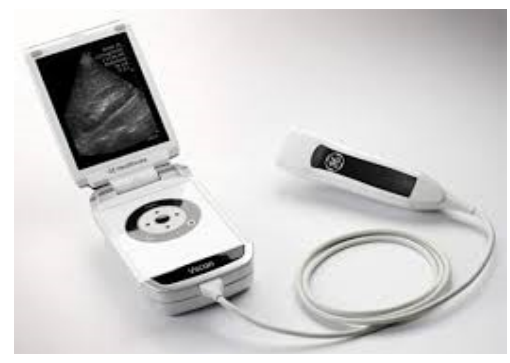

Fig. 1. Handheld ultrasound system from GE.

proposed in [2] to address emergency medical applications. In many countries, doctors are not willing to serve in rural areas due to unavailability of proper infrastructure for healthcare. Telemedicine can reduce the geographical separation between the patients and provide better remote healthcare.

Diagnosis in remote areas using handheld ultrasound machine with communication module is done by transmitting the acquired ultrasound images to cloud, sonographer can access the data in the cloud from any location and send the diagnosis report regarding the images.

The person who acquires and do analysis on the ultrasound images are called sonographers, as per rural health statistics in India 2012, there is a requirement of 4833 sonographers at Community Health Centers (CHC), where only 2314 sonographer positions (less than 50\%) are filled [3]. Due to lack of sonographers, handheld ultrasound machines at remote areas are operated by semi-skilled clinicians. Ultrasound scanning involves high subjectivity and probability of acquiring ultrasound images which are not useful for diagnosis is high. Transferring all the acquired images from ultrasound scanning machines will increase the data in cloud; this will increase the diagnosis time of the sonographer as they have to analyse all the images available in the cloud.

Due to advancements in telemedicine, more number of healthcare devices are getting connected to Internet of Things (IoT) and according to Morgan Stanley analysis 75 Billion devices will be connected to IoT by 2020 [4], which leads to hyper connectivity and network congestion due to transmission of large medical data. Transmission of redundant/invalid medical data drains the battery of handheld ultrasound machines, hence there is a need to avoid transmitting of redundant/invalid medical data getting into cloud, which 
avoids congestion in the network traffic and also reduces the medication time as the sonographer has to diagnose on limited number of images.

The rest of the paper is organized as follows, section II briefly explains the characteristics of ultrasound images, problem statement and basis for image classification. Algorithm implementation for validating the ultrasound images for diagnosis is explained in section III. In section IV, results are discussed and section $\mathrm{V}$ concludes the paper.

\section{Characteristics OF Ultrasound IMAGES}

Ultrasound scanning involves high subjectivity and organs shape and size will vary depending upon the position and angle of the transducer probe. The shape and size of organ also varies from patient to patient which makes difficult for sonographers to conform the presence of organs in the image. The signal to noise ratio of ultrasound images is poor and is effected with a special type of noise called speckle noise [5]. Speckle is a multiplicative noise which appears as granular and small snake like structures in the ultrasound image. Speckle noise delineates the edges and also conveys echogenicity of tissues. Speckle patterns and echo structure of tissues cannot be differentiated and speckles have to be preserved for organ detection and tracking like applications. Ultrasound image generated using K-wave tool box in Matlab [6] is shown in Fig. 2. The K-wave parameter specifications used for generating the ultrasound image for the template are shown in Table I. The small snake like structures in the image corresponds to speckle patterns; these patterns are useful for detecting the property of a tissue. The texture features of speckle patterns will be useful for image classification. The uniform speckle patterns present all over the image does not convey any information regarding organs. The homogeneity of texture is considered as useful feature for detecting invalid images. Ultrasound images also affected with acoustic shadowing due to occlusion of bones, stones which has high attenuation to acoustic signal preventing organs to display on the screen.

To reduce the data in cloud, an algorithm is developed to validate the ultrasound image into valid and invalid for transmission based on the organ information present in the image. The ultrasound images useful for diagnosis is

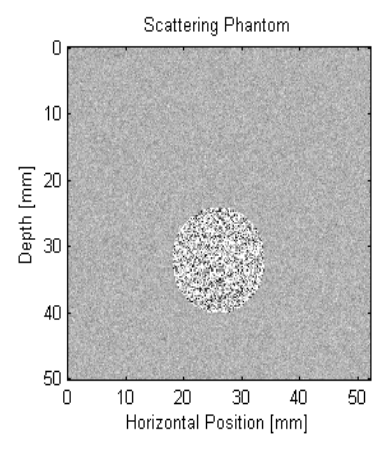

(a)

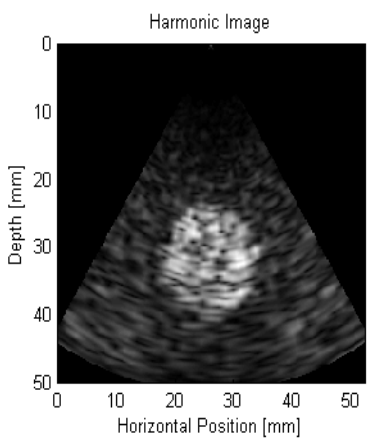

(b)
Fig. 2. (a) Phantom template. (b) Software generated ultrasound image.
TABLE I

K-WAVE PARAMETERS USED TO SIMULATE THE ULTRASOUND IMAGE SHOWN IN FIG. 2(A).

\begin{tabular}{||l|l||}
\hline Transducer width & $14.1593 \mathrm{~mm}$ (64 grid points) \\
\hline Number of elements & 64 \\
\hline Number of active elements & 64 \\
\hline Element width & $221.2389 \mathrm{um}$ (1 grid points) \\
\hline Sound speed & $1540 \mathrm{~m} / \mathrm{s}$ \\
\hline Focus distance & $30 \mathrm{~mm}$ \\
\hline Elevation focus distance & $30 \mathrm{~mm}$ \\
\hline
\end{tabular}

classified as valid images and images which does not convey any information regarding organs are considered as not useful for diagnosis and classified as invalid images.

\section{A. Valid ultrasound images}

Valid ultrasound images consists of significant structures like valleys, peaks, edges, inhomogeneity in echo structure etc., are shown in Fig. 3. Valid ultrasound images includes cardiac, liver, kidney, fetus organs etc. Homogeneous structures will not be present all over the image and it is considered as useful feature for image classification.

\section{B. Invalid ultrasound images}

Invalid ultrasound images have uniform echo structures in the image. Significant structures like peaks and valleys which infer about organs are absent in the ultrasound images. These sort of images will not convey any information about organs and are not useful for diagnosis. The invalid ultrasound images not useful for diagnosis are shown in Fig. 4.

\section{AlgORITHM IMPLEMENTATION}

Ultrasound images are classified into valid and invalid images based on the texture features of the image. Ultrasound

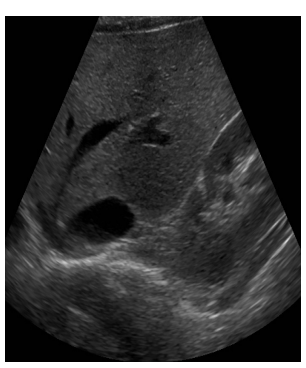

Liver

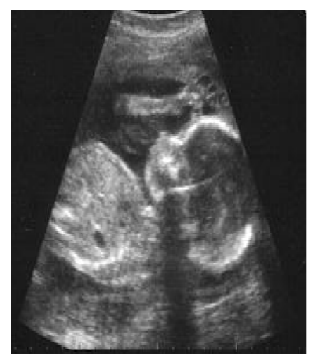

Fetus

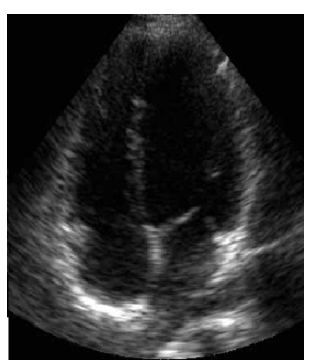

Cardiac

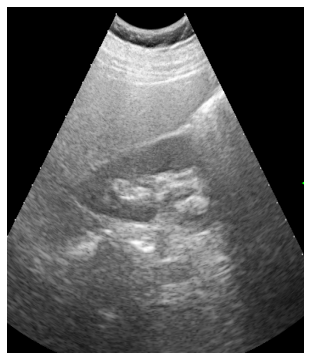

Kidney
Fig. 3. Valid ultrasound images. 

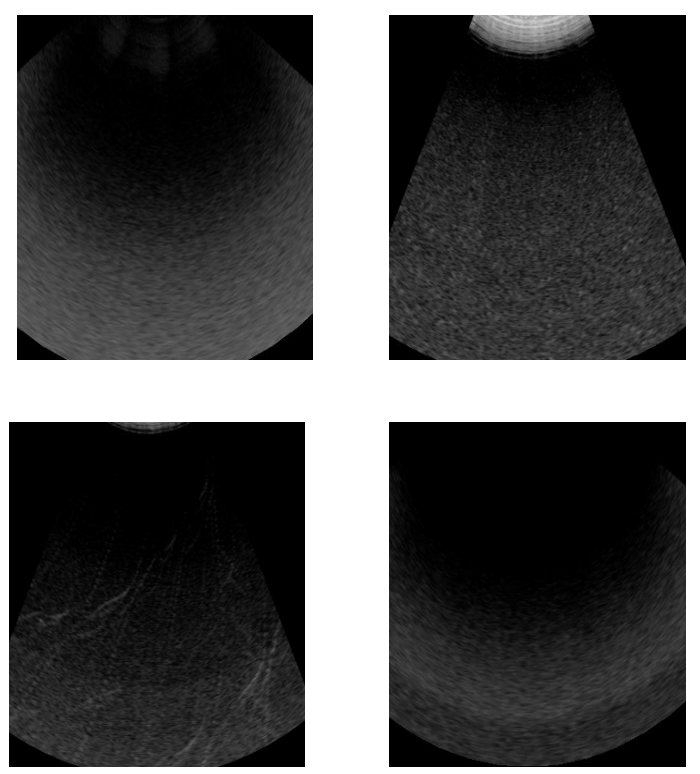

Fig. 4. Invalid ultrasound images.

image depends upon the type of excitation and transducer probe used for scanning the organ. The selection of transducer probe depends upon the scanning of a particular organ. Generally ultrasound images appears as square and sector images. In square ultrasound images, the organ information is present all over the image. In sector ultrasound images the organ information is present similar to a cone shape. All images are cropped manually to remove the labels and markings on the ultrasound images, which is an artifact for feature extraction. Image validation for diagnosis is done in two stages. First stage involves detecting region of interest automatically in the image and second stage involves feature extraction and classification as shown in Fig. 5. The flowchart representation of ultrasound image validation for diagnosis is shown in Fig. 6.

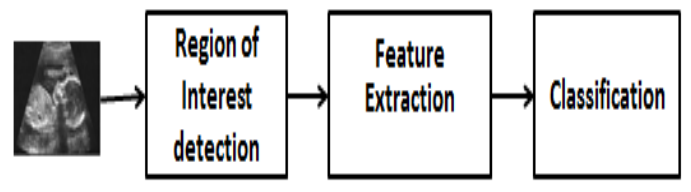

Fig. 5. Block diagram representation of image validation.

\section{A. Region of interest detection}

The objective of region of interest (ROI) detection is to look for the region in an image where it has maximum information about organ. The imaging plane of ultrasound scanning embeds the information in cone shaped image. A circle with appropriate radius can cover maximum information present in the image. The center and radius of the circle is selected empirically and these may change depending on the ultrasound scanning machine. The radius and center of a circle is selected in such a way that ROI is enclosed within cone shaped ultrasound imaging plane.

The image tested for validation is normalized by resizing it to a standard $512 \times 512$ size. $512 \times 512$ gives the total number of pixels present in the image and also signifies number of rows and columns present in the image. The ultrasound image is normalized to overcome the size differences in the image. The image is scanned vertically (along $\mathrm{X}$-axis) to detect the starting and ending pixels with nonzero intensity values. Let the index of starting and ending nonzero pixel intensity along the $\mathrm{X}$-axis be $\left(x_{i}, y_{1}\right),\left(x_{j}, y_{1}\right)$ and $i, j \in[1$, 512]. 1 is minimum and 512 is maximum value $i, j$ can take. The midpoint of starting and ending nonzero pixel value is the required column of an image. The midpoint is computed by

$$
(x, y)=\left(\frac{x_{i}+x_{j}}{2}, \frac{y_{1}+y_{1}}{2}\right)
$$

The required row number is chosen as 307 ( 0.6 times the 512). A circle having a radius of 200 pixels being $(x, 307)$ index as center is considered as region of interest. The pixel indexes which satisfy the locus of the circle equation given in (2) are considered for feature extraction and it will cover almost all pixels present in the ultrasound imaging plane.

$$
\left(x_{i}-x\right)^{2}+\left(y_{i}-307\right)^{2} \leq 200^{2}
$$

The ROI detection of an ultrasound image is shown inside the red circular region in the Fig. 7. The center of circle is marked with ' $x$ ' mark and radius of circle with length 200 pixels is considered as region of interest.

\section{B. Feature extraction}

The pixel intensity values inside the region of interest are only considered for feature extraction. The first order features including mean, variance, skewness and kurtosis are computed using the following equations.

$$
\begin{gathered}
\mu=\left(1 / W^{2}\right) \sum_{m=-W / 2}^{W / 2} \sum_{n=-W / 2}^{W / 2} I(i-m, j-n) \\
\sigma^{2}=\left(1 / W^{2}\right) \sum_{m=-W / 2}^{W / 2} \sum_{n=-W / 2}^{W / 2}(I(i-m, j-n)-\mu)^{2} \\
S=\left(1 / W^{2}\right) \sum_{m=-W / 2}^{W / 2} \sum_{n=-W / 2}^{W / 2}(I(i-m, j-n)-\mu)^{3} \\
K=\left(1 / W^{2}\right) \sum_{m=-W / 2}^{W / 2} \sum_{n=-W / 2}^{W / 2}(I(i-m, j-n)-\mu)^{4}
\end{gathered}
$$

$I(i-m, j-n)$ over the double summation in (3), (4), (5) and (6) takes all the pixel intensities within the region of interest.

Mean $(\mu)$ gives the average intensity of the pixels in the ROI, variance $\left(\sigma^{2}\right)$ gives the spread of pixel distribution, skewness (S) gives the measure of symmetry and kurtosis (K) gives the sharpness of pixel distributions. 
TABLE II

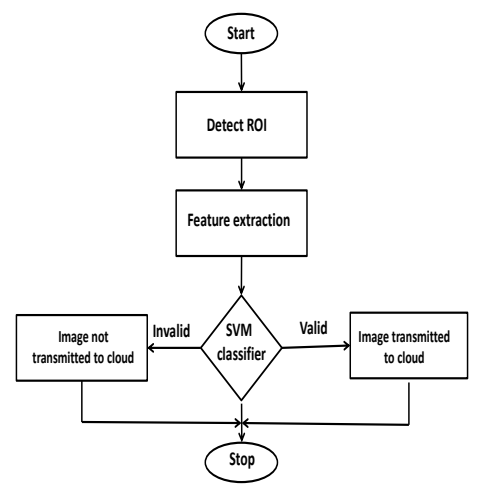

Fig. 6. Flow chart representation of image validation for transmitting to cloud.

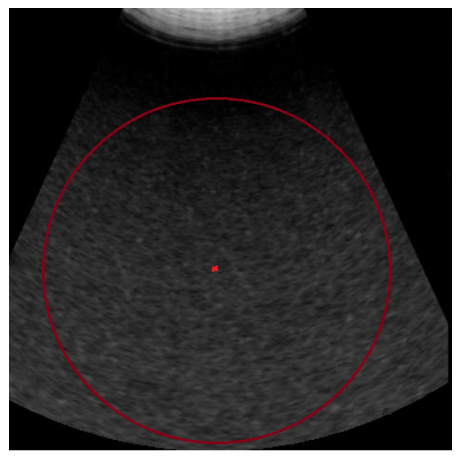

Fig. 7. Region of interest detection.

\section{Data acquistion}

Ultrasound image database is acquired from Nitya diagnostic center, Hyderabad, India. Ultrasound images regarding valid and invalid for diagnosis are confirmed with three independent sonographers. Ultrasound image database consisted of 90 invalid and 150 valid sector ultrasound images.

Linear kernel Support Vector Machine (SVM) classifier [7] is used to classify the images into valid and invalid images for diagnosis. A linear kernel SVM classifier is initially trained with features of valid and invalid images. The algorithm will automatically detect the region of interest from the image and compute the features from ROI. These features are fed to the trained SVM classifier to know the information present in the image for diagnosis. The algorithm is realized using Matlab software.

\section{RESULTS}

The image validation algorithm is tested with 60 valid images and 80 invalid images. The recognition performance of the algorithm is tested by plotting the confusion matrix shown in Table II. The algorithm recognized 3 valid images as invalid images when tested with 60 valid images, resulting 95\% (57 out of 60) recognition efficiency for valid images. All the 3 wrongly classified images belong to liver ultrasound, ambiguity in recognizing the liver image is due
CONFUSION MATRIX FOR AUTOMATIC ULTRASOUND IMAGE

VALIDATION.

\begin{tabular}{c|c|c|c|}
\multicolumn{2}{c}{} & \multicolumn{2}{c}{ Predicted class } \\
\cline { 3 - 4 } \multicolumn{1}{c|}{} & Valid & Invalid \\
\cline { 2 - 4 } & Valid (60) & 57 & 3 \\
\cline { 2 - 4 } Actual class & Invalid (80) & 5 & 75 \\
\cline { 2 - 4 } & &
\end{tabular}

to its echo structure which is similar to invalid ultrasound images. 5 invalid images are wrongly classified as valid images resulting a recognition efficiency of $93.75 \%$ ( 75 out of 80) for invalid images. The overall recognition of the algorithm is $94.28 \%$ (132 out of 140).

\section{CONCLUSION}

The amount of data transmitted to cloud will be significantly reduced when more number of portable ultrasound devices deployed in the field. Avoiding transmission of invalid images reduces the data in cloud thus improving the medication time, battery of the chargeable ultrasound device and also avoids network traffic. The future work includes the detection of invalid slices in ultrasound video which can drastically reduce the amount of ultrasound data being transmitted to cloud.

\section{REFERENCES}

[1] Kyu Cheol Kim, Min Jae Kim, Hyun Suk Joo, Wooyoul Lee, Changhan Yoon, Tai-kyong Song, and Yangmo Yoo, "Smartphone-based portable ultrasound imaging system: A primary result," Ultrasonics Symposium (IUS), IEEE International, vol., no., pp.2061,2063, 2125 July 2013.

[2] Chiang, A.M, Chang P.P and Broadstone S.R, "PC-based ultrasound imaging system in a probe," Ultrasonics Symposium, 2000 IEEE , vol.2, no., pp. 1255,1260 vol.2, Oct 2000 .

[3] Statistics Division Ministry of Health and Family Welfare, Government of India, "Rural Health Statistics in India 2012," March, 2012.

[4] http://www.businessinsider.in/Morgan-Stanley-75-BillionDevices-Will-Be-Connected-To-The-Internet-Of-Things-By2020/articleshow/23426604.cms

[5] C. B. Burckhardt, "Speckle in ultrasound B-mode scans," IEEE Trans Ultrason., vol.SU-25, pp. 1-6, Jan 1978.

[6] B. E. Treeby and B. T. Cox, "k-Wave: MATLAB toolbox for the simulation and reconstruction of photoacoustic wave-fields," J. Biomed. Opt., vol. 15, no. 2, p. 021314, 2010.

[7] Tong Zhang, "An Introduction to Support Vector Machines and Other Kernel-Based Learning Methods," AI Magazine, Volume 22, Number 2,2001 . 